DOI:

$10.1038 / \mathrm{nrm} 2456$

\title{
Roundworm joins the piRNA club
}

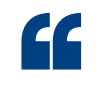

.... a function for Piwi and 21U-RNAs in the germline.
The Piwi subclass of Argonaute proteins associate with 24-30-nucleotide RNAs called Piwi-interacting RNAs (piRNAs) in the fruitfly, zebrafish, mouse and rat. Three new studies report the identification of a related class of 21nucleotide RNAs, $21 \mathrm{U}$-RNAs, as the piRNAs of Caenorhabditis elegans and suggest a function for Piwi and 21U-RNAs in the germ line.

C. elegans has two Piwi proteins, PRG-1 and PRG-2. Both genes were mutated to investigate the previously proposed role of Piwi proteins in the germ line. Data by Wang and Reinke suggest that the main reason for reduced fertility in the prg- 1 mutant is a defect at the very late stage of spermatogenesis. By contrast, the other two teams conclude that Piwi has a broader role in both male and female gametes.

So, which class of small RNAs in C. elegans represents the piRNAs?
21U-RNAs share several features with the piRNAs of the fruitfly and mammals that prompted the teams to test whether these short RNAs were dependent on Piwi. Das et al. generated libraries of small RNAs from wild-type and double mutants (referred to as piwi mutants), and found that 21U-RNAs were either absent or underrepresented in the piwi-mutant library compared with the wild-type library. Wang and Reinke tested the expression levels of two 21U-RNAs, which were reduced in prg-1 mutants. Batista et al. and Das et al. carried out high-throughput sequencing analysis of small-RNA populations from $\mathrm{prg}-1$ and $\mathrm{prg}-2$ mutants and observed a dramatic depletion of 21U-RNAs in the mutants compared with the wild-type worm. Batista et al. also showed that PRG-1 immunoprecipitates were strongly enriched for 21U-RNAs. These findings suggest that PRG-1 is

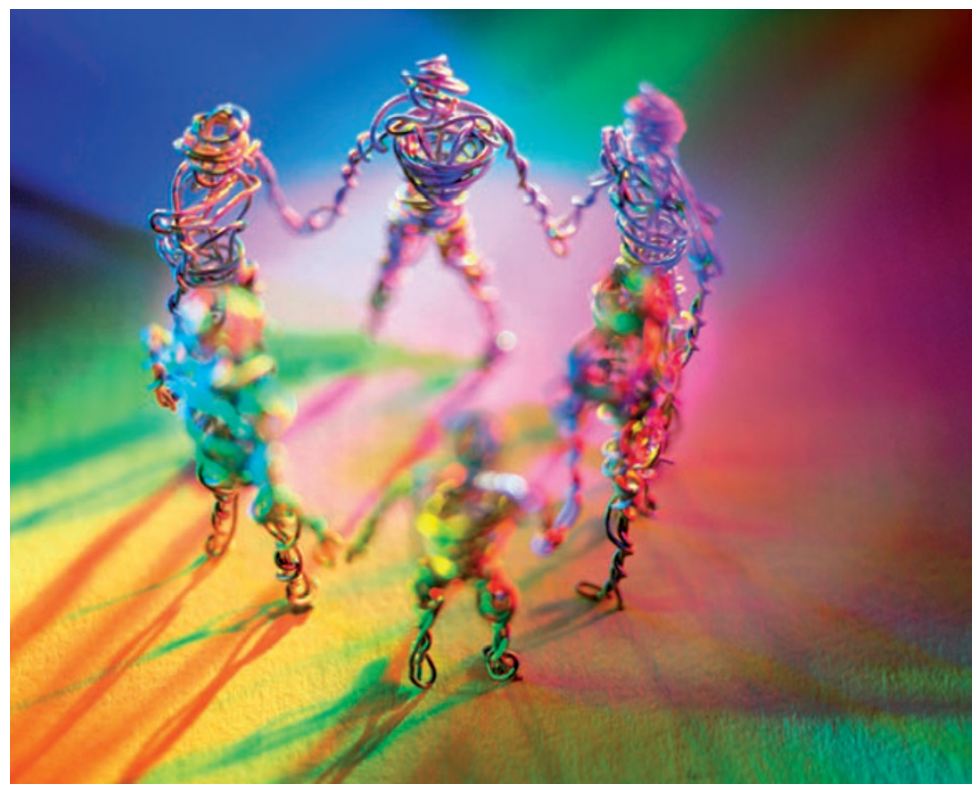

likely to be required for the accumulation of the entire class of 21U-RNAs.

The Batista et al. and Das et al. teams showed that expression of 21U-RNAs and PRG-1 is restricted to the male and female germ line. But how does PRG-1 function during germline development? When comparing dissected gonads from wild-type and prg-1 mutant males, Wang and Reinke found that the loss of prg-1 causes a marked reduction in the expression of a subset of spermatogenesis-specific transcripts. Batista et al. noticed a significant upregulation of transposon Tc3 mRNA in prg- 1 mutants, which was also observed by Das et al., who further showed a large increase in Tc3 transposition in these mutants - suggesting a role for Piwi in transposon silencing.

It seems likely that Piwi and 21U-RNAs function in gene regulation, in addition to regulating transposon mobility, during germline development. However, perhaps the most intriguing aspect of piRNAs in worms is their lack of obvious targets; among the $>15,000$ piRNA species that have been detected only one targets a transposon.

Arianne Heinrichs

ORIGINAL RESEARCH PAPERS Wang, G. \& Reinke, V. A. C. elegans Piwi, PRG-1, regulates 21U-RNAs during spermatogenesis. Curr. Biol. 18 861-867 (2008) | Batista, P. J. et al. PRG-1 and 21U-RNAs interact to form the piRNA complex required for fertility in C. elegans. Mol. Cell 19 June 2008 (doi:10.1016/j.molcel.2008.06.002)| Das, P. P. et al. Piwi and piRNAs act upstream of an endogenous siRNA pathway to suppress Tc3 transposon mobility in the Caenorhabditis elegans germline. Mol. Cell 19 June 2008 (doi:10.1016/j.molcel.2008.06.003) FURTHER READING Stefani, G. \& Slack, F. J. Small non-coding RNAs in animal development. Nature Rev. Mol. Cell Biol. 9, 219-230 (2008)| Hutvagner, G. \& Simard, M. J. Argonaute proteins: key players in RNA silencing. Nature Rev. Mol. Cell Biol. 9, 22-32 (2008) 